\title{
Observer Organization
}

National Cancer Institute

\section{Source}

National Cancer Institute. Observer Organization. NCI Thesaurus. Code C69246.

The affiliated organization of an observer. 